\title{
LA QUALITÉ DU LOGEMENT COMME VARIABLE ENVIRONNEMENTALE : L'EXEMPLE DE LA RÉGION URBAINE DE LIÈGE (WALLONIE)
}

\author{
Zoé Lejeune et al. \\ Métropolis I Flux \\ 2012/3 - N 89-90 \\ pages 30 à 45
}

ISSN 1154-2721

Article disponible en ligne à l'adresse:

http://www.cairn.info/revue-flux-2012-3-page-30.htm

Pour citer cet article :

Lejeune Zoéet al., « La qualité du logement comme variable environnementale : l'exemple de la région urbaine de Liège (Wallonie) »,

Flux, 2012/3 № 89-90, p. 30-45.

Distribution électronique Cairn.info pour Métropolis.

(c) Métropolis. Tous droits réservés pour tous pays.

La reproduction ou représentation de cet article, notamment par photocopie, n'est autorisée que dans les limites des conditions générales d'utilisation du site ou, le cas échéant, des conditions générales de la licence souscrite par votre établissement. Toute autre reproduction ou représentation, en tout ou partie, sous quelque forme et de quelque manière que ce soit, est interdite sauf accord préalable et écrit de l'éditeur, en dehors des cas prévus par la législation en vigueur en France. II est précisé que son stockage dans une base de données est également interdit. 


\title{
La qualité du logement comme variable environnementale: I'exemple de la région urbaine de Liège (Wallonie)
}

\author{
Zoé Lejeune \\ Thomas Chevau \\ Jacques Teller
}

L e logement constitue un élément fondamental de la qualité de vie des populations. En Wallonie, l'article 2 du Code du Logement établit que "les autorités publiques mettent en œuvre le droit à un logement décent en tant que lieu de vie, d'émancipation et d'épanouissement des individus et des familles ». L'accès à un logement de qualité y reste cependant un important vecteur de différenciation sociale. On observe ainsi que 3,4\% des ménages wallons sont en attente d'un logement social, dont plus de $75 \%$ sont considérés en état de précarité (1) (IWEPS, 2007, p. 112). Le "parc social de fait", comme il est appelé en Wallonie (c'est-à-dire le parc de logement privé de mauvaise qualité loué à des populations précarisées n'ayant pas accès au logement social faute d'offre suffisante), constitue la principale réponse à cette demande. De nombreux problèmes se posent quant à ce type de logements: souvent de mauvaise qualité, suroccupés, vétustes, etc., ils sont occupés par des personnes qui sont « rejetées » des autres segments du secteur du logement (De Decker, Laureys, 2007, pp. 4-5). À l'instar de ce que l'on observe dans le nord de la France, le parc de logements est très ancien en Wallonie: plus de $50 \%$ des logements ont été construits avant 1945 (Dujardin et al., 2010, p. 65). Une partie significative de ce parc est établie à proximité directe d'anciens sites de production industrielle, qui sont parfois toujours en activité.

Nous proposons ici de considérer les attributs du logement (superficie, salubrité, chauffage central, taux d'occupation, isolation, etc.) comme une variable environnementale susceptible de renforcer les inégalités socioéconomiques, au même titre que l'exposition au bruit, à la pollution atmosphérique ou l'accès aux aménités urbaines. Nous envisageons l'impact de cette variable au sens large, non pas uniquement sur la santé mais, plus généralement, sur la qualité de vie au sens large (2). Ceci revient à dire que nous considérons que la capacité des ménages à contrôler les qualités intrinsèques (Geymard, 2004) du logement, à savoir sa taille, son type de chauffage, son degré de salubrité, est très inégalement distribuée spatialement à l'intérieur des villes. En effet, on ne change pas facilement la superficie d'un logement; il n'est pas toujours possible ni raisonnable, pour un locataire, d'isoler le logement qu'il occupe.

L'incidence du logement sera mise en lien avec deux types de services ou aménités urbains qui jouent également un rôle important pour la qualité de vie: I'accessibilité par les alternatives à la voiture et l'accès à des espaces verts. Notre étude consiste à montrer comment ces inégalités environnementales renforcent, compensent ou sont déconnectées des inégalités socioéconomiques et à spatialiser ces processus dans une ville de Wallonie, la région urbaine de Liège, marquée par un passé industriel encore très prégnant.

Nous développerons, dans un premier temps, la question de la qualité du logement et la manière dont celle-ci est abordée dans le cadre de la littérature relative aux inégalités environnementales et à la justice environnementale. Dans un second temps, nous présenterons notre cas d'étude, à savoir la région urbaine de Liège et les principales caractéristiques des centres urbains wallons du sillon industriel. Nous introduirons ensuite l'indice socioéconomique qui nous a servis de base pour le croisement avec la qualité du logement, conçue comme vecteur d'inégalités environnementales. Finalement, un dernier point abordera les liens avec deux types d'aménités urbaines, à savoir, d'une part, l'accessibilité par les alternatives à la voiture et, d'autre part, l'accès aux espaces verts.

\section{LA QUESTION DU LOGEMENT DANS L'ANALYSE DES INÉGALITÉS ENVIRONNEMENTALES}

La définition la plus commune donnée en Europe (3) aux inégalités environnementales affirme qu'il s'agit d'une « forme spéci- 
fique d'inégalité sociale qui concerne soit l'exposition aux pollutions ou aux risques, soit l'accès à la nature ou aux aménités urbaines ou rurales, soit encore la capacité d'action des citoyens »(Theys, 2007, p. 25). La plupart des études portant sur les inégalités environnementales traitent des qualités extrinsèques du logement et du lieu de vie, à savoir l'environnement au sens large où vivent les populations souvent les plus défavorisées: pollution atmosphérique, proximité d'installations polluantes, d'infrastructures de transport bruyantes, etc.

Sous le concept de justice environnementale, la recherche s'est développée dès les années 1980 aux États-Unis (Boulder Wenz, 1988; Bullard, 1990; EPA, 2004; etc.) sur les inégalités subies par les populations défavorisées ou issues d'une minorité ethnique, en termes de localisation d'installations polluantes au sens large. La justice environnementale nord-américaine s'est développée en tant qu'objet de recherche spécifique visant à apporter une plus-value à l'étude des inégalités sociales, $d^{\prime}$ 'une part, et en tant que mouvement social de contestation et de défense des droits des minorités et des populations pauvres (Matsuoka, 2001), d'autre part. L'approche des discriminations par le logement (housing discrimination and residential segregation) y constitue un domaine de recherche des études urbaines, tout comme le concept " LULU » (locally unwanted land use), qui se réfère aux réponses sociales négatives apportées aux projets d'installations polluantes. Beaucoup de travaux nord-américains prennent les zones urbaines comme espaces de réflexion sur la justice environnementale. Par rapport à ces divers constats et aux politiques publiques mises en place suite à la création de l'Environmental Protection Agency aux ÉtatsUnis, la recherche et les politiques restent quant à elles très parcellaires en Belgique sur ces thématiques. Ces dernières sont plutôt axées sur les discriminations subies par les populations pauvres plutôt qu'appartenant à des minorités. On peut ainsi s'étonner du fait que cette notion ne soit encore que peu intégrée au sein des politiques publiques en Wallonie.

Nous proposons ici d'étudier les relations entre populations socioéconomiquement défavorisées et nuisances environnementales, mises en lumière au travers du logement, témoignant d'inégalités spatiales entre populations à l'échelle urbaine.

Certains auteurs (Faburel, Gueymard, 2008) considèrent que l'on peut distinguer deux visions de l'environnement, la première plus axée sur l'environnement physique et naturel et la seconde, plus dynamique, sur l'environnement créé par
I'homme, dont l'habitat est un aspect important. La séparation entre ces deux approches a conduit à une altérité homme/nature et à une vision non intégrée de leur cohabitation, qui expliquerait notamment la mise en place de politiques axées sur I'une ou l'autre de ces visions (Faburel, Gueymard, 2008, pp. 159-160). " Le cadre théorique permettant de croiser environnement et justice sociale demeure fragile, comme si les deux champs de réflexion s'étaient mutuellement ignorés " (Blanchon et al., 2009, p. 42). Ces auteurs constatent ainsi que I'« on manque de cadre conceptuel pour penser l'environnement et les inégalités sociales, et définir une éthique de l'environnement » (ibid., p. 45). II faudrait dès lors réconcilier ces deux cultures afin de pouvoir répondre aux enjeux des inégalités environnementales et sociales en milieu urbain (Theys, 2002, p. 6), dans une perspective de développement durable et intégré. Les valeurs environnementales (pollution, aménités, etc.) sont perçues également de manière très différente $d^{\prime}$ un groupe ou d'un individu à l'autre, ce qui rappelle la subjectivité dans l'appréhension de ces désaménités. En effet, la perception et l'utilisation de l'environnement sont variables selon les groupes sociaux.

Laigle et Oehler (2004) envisagent quatre grandes catégories d'inégalités environnementales. Ces auteurs identifient notamment les inégalités " d'accès à l'urbanité et à un cadre de vie de qualité ", dont le logement est un élément central, mais également "les inégalités liées à l'héritage ". Ces dernières, liées au développement " historique " des territoires urbains, peuvent intégrer des facteurs de localisation mais aussi des éléments relatifs au logement. La qualité et le confort du logement sont pour partie liés à sa période de construction. Notre approche vise notamment à « repérer les vulnérabilités sociales et environnementales héritées des modes de développement antérieurs » (Zaccaï, 2007), expliquant notamment l'état du bâti dans les quartiers anciens et centraux des villes du sillon industriel wallon. Le logement et ses qualités peuvent ainsi s'insérer dans deux des catégories proposées. En effet, contrairement à une approche des inégalités environnementales plus classique axée sur l'impact des nuisances provenant de l'environnement extérieur au logement, l'approche ici choisie d'étudier ces inégalités par la qualité du logement permet de questionner la typologie proposée par Laigle et Oehler (2004).

Finalement, " la définition minimale des inégalités environnementales, c'est-à-dire le seul croisement descriptif des dotations et/ou pressions environnementales et des caractéristiques 


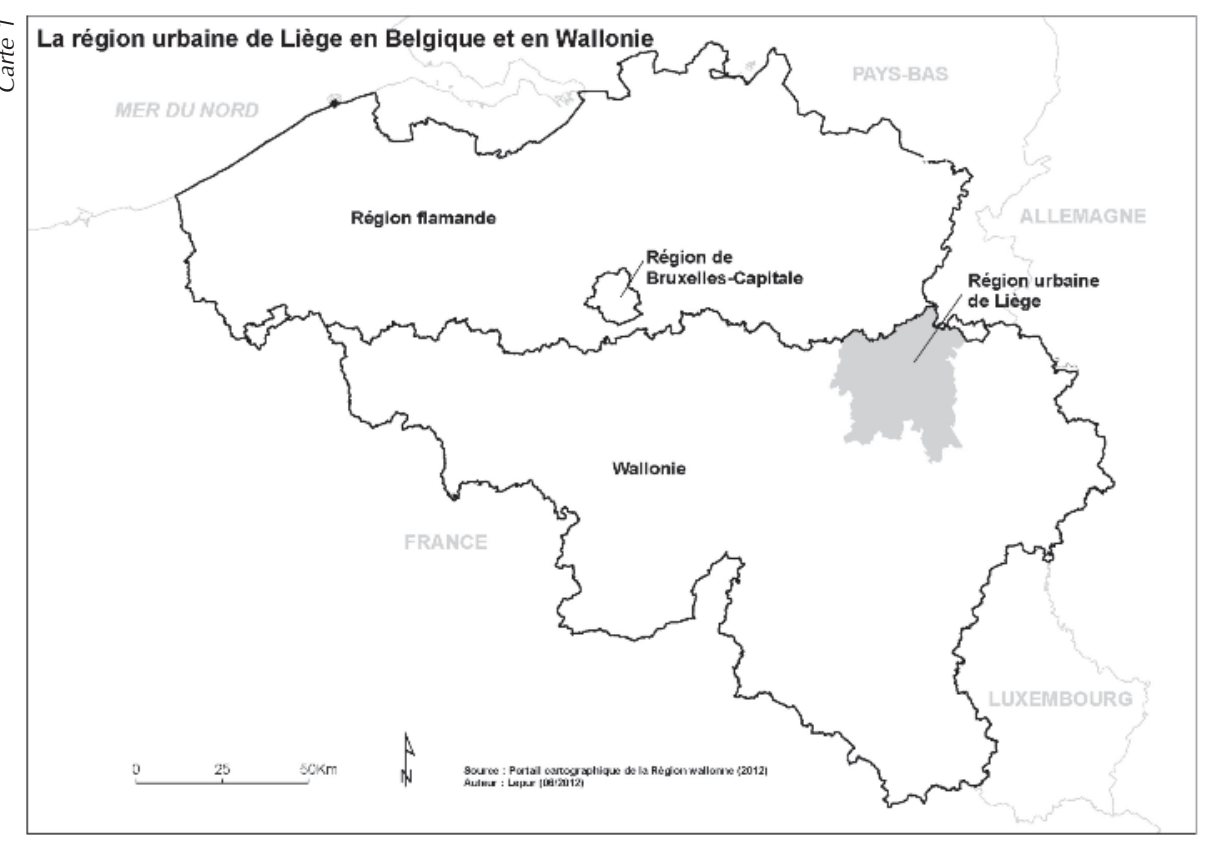

gistraient les moins bons scores en termes de qualité de l'environnement perçu (Vanneste et al., 2007, p. 153). Les choix résidentiels des populations sont fortement contraints et mélangent des éléments relevant des qualités intrinsèques et extrinsèques des lieux de vie.

\section{LES INÉGALITÉS SOCIOÉCONOMIQUES DANS LA RÉGION URBAINE DE LIÈGE}

Nous nous intéressons ici à la relation entre les qualités intrinsèques du logement (superficie, type de chauffage, etc.) et les disparités socioéconomiques. Nous établirons ensuite un parallèle entre la relation observée dans ce cadre et celle que l'on peut observer

sociales des espaces, ne suffit pas à rendre compte non seulement des processus à l'œuvre, mais aussi de la multiplicité des facteurs qui interviennent dans leur construction. [...] Ils [les exemples d'inégalités environnementales] interrogent en amont de concert les relations que les inégalités environnementales entretiennent avec la fabrique et la gestion de la ville » (Faburel, 2008, p. 114).

Nous nous concentrons dans cette étude sur une approche des inégalités environnementales par l'entrée du logement, donnant toute son importance au cadre de vie « intérieur » des populations. Le logement et sa qualité intérieure constituent en effet « un élément central de la satisfaction résidentielle et des choix de localisation [et] peut [dès lors] contrebalancer la qualité de l'environnement immédiat, dans un champ complexe d'interférences » (Emelianoff, 2010, p. 190). Plusieurs études montrent d'ailleurs que « le prix du logement s'avère surdéterminé par ses attributs internes, beaucoup plus que par ses attributs extérieurs »(Geymard, 2004, p. 15). Il est toutefois difficile « d'isoler le poids de la qualité du logement physique de celle de l'environnement social qui est également recherché et qui généralement est fortement corrélé à la qualité de l'environnement physique » (ibid., p. 16). La dernière enquête socioéconomique menée en Belgique en 2001 s'intéressait notamment à l'environnement du logement, par le prisme de la perception qu'en avaient les enquêtés. Les agglomérations y enre- pour deux types de services urbains : I'accessibilité par les transports en commun et l'accès aux espaces verts. Ces deux services ont été choisis car ils permettaient d'étudier les inégalités environnementales à plusieurs échelles urbaines, allant du logement à I'agglomération. Cette étude est menée sur la région urbaine de Liège que nous présentons brièvement ci-après.

Située dans le sillon industriel traversant la région d'ouest en est, Liège est une des deux " grandes » villes de Wallonie. Caractérisée par la présence historique d'une importante industrie sidérurgique et métallurgique, la ville connaît des problèmes de reconversion économique de son bassin industriel et de précarisation générale de ses quartiers centraux, liés à la périurbanisation tant résidentielle que commerciale et économique. La région urbaine de Liège (voir les cartes 1 et 2) a été délimitée sur la base du découpage proposé par Van der Haegen et al. (1996) pour la Belgique (4). Celle-ci regroupe trente-cinq communes sur un territoire de $1076 \mathrm{~km}^{2}$, pour environ 650000 habitants, sans qu'il n'y ait de véritable coopération institutionnelle pour piloter son développement, du type de celle que l'on rencontre dans les communautés urbaines françaises. En effet, aucune politique spécifique n'est à l'heure actuelle menée à l'échelle supra-communale en Wallonie (hormis l'une ou l'autre expérience spontanée telle que la mise en place d'un plan urbain de mobilité à l'échelle de l'agglomération liégeoise, par exemple). 
Les populations les plus défavorisées en Wallonie et a fortiori dans la région urbaine de Liège sont concentrées dans les quartiers centraux des villes, alors que les populations aisées leur préfèrent la périphérie. Ce phénomène de périurbanisation engendre en Belgique « un fort affaiblissement de la base fiscale des communes urbaines centrales » (Vandermotten et al., 2006, p. 7), suite à la concentration de populations plus précarisées en leur sein. Ces communes urbaines ne peuvent désormais supporter seules les charges que suppose I'utilisation de ces services et équipements par des populations n'y résidant pas.

Les populations plus précarisées se concentrent ainsi essentiellement dans les quartiers anciens et centraux, ainsi qu'en première couronne des villes, plutôt sur le modèle anglo-américain que français (ibid., p. 8).

Cette situation est clairement observable à travers les cartes 3 et 4, qui illustrent la variation de l'indice socioéconomique au sein de la région urbaine liégeoise. Cet indice est construit sur base de l'agrégation de six variables détaillées dans le tableau 1.

Pour calculer cet indice, nous avons normalisé chaque variable de manière à lui accorder une valeur située dans un intervalle de 0 à 1 , en considérant, par convention, que pour chacune des variables, 0 correspond à l'indice socioéconomique le plus défavorable et 1 au plus favorable. L'indice du quartier correspond à la moyenne des valeurs standardisées des six variables. Les valeurs minimales et maximales ont été extraites de l'échantillon.

$$
\mathrm{I}_{\mathrm{i}}=\sum_{j=1}^{6} \frac{I i j-\operatorname{Min} I j}{[\operatorname{Max} I j-\operatorname{Min} I j]}
$$

$\mathrm{I}_{\mathrm{i}}=$ indice socioéconomique du secteur statistique $\left(\mathrm{i}_{\mathrm{i}}\left[\begin{array}{lll}0 & \ldots & 1\end{array}\right]\right)$. $\mathrm{I}_{\mathrm{ij}}=$ valeur de la variable (1 à 6) constitutive de l'index pour le

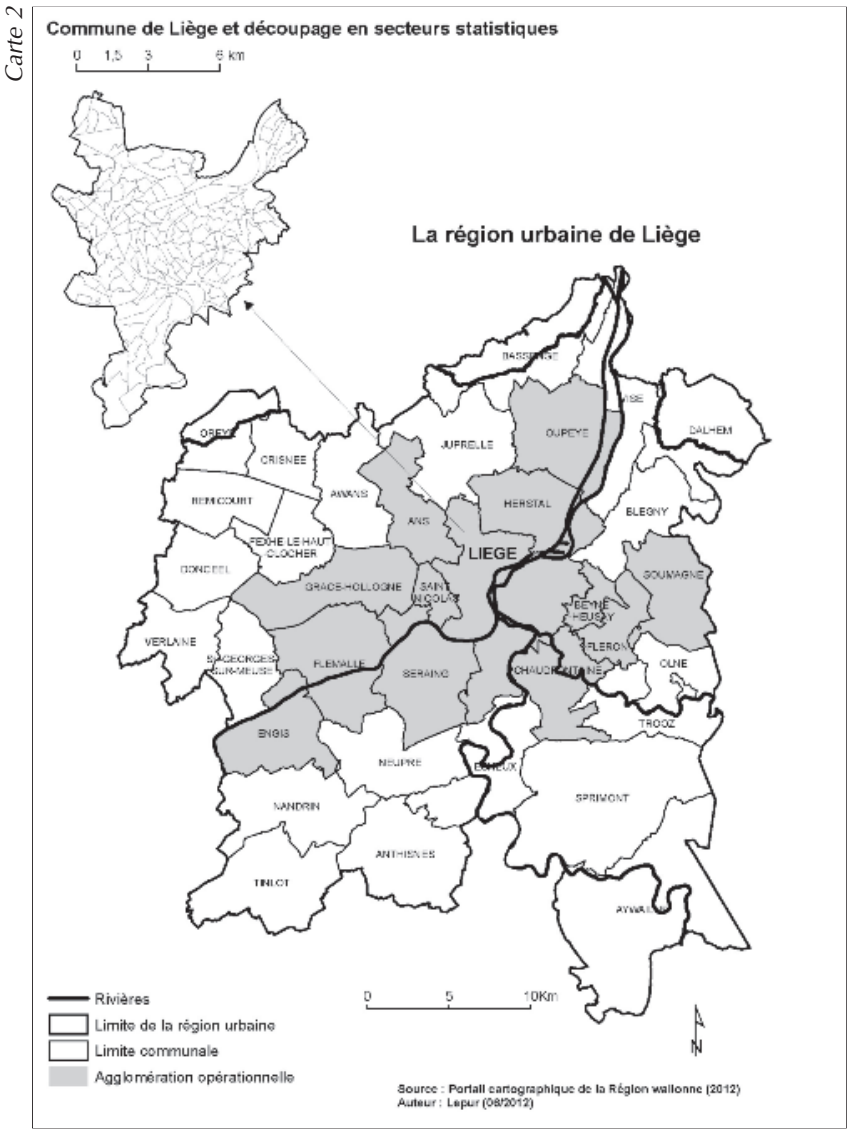

quartier/secteur statistique i. Les valeurs sont ordonnées du minimum au maximum.

Max. $\mathrm{I}_{\mathrm{j}}=$ valeur maximale de la variable (1 à 6) pour l'ensemble des quartiers de la région urbaine, après élimination des valeurs extrêmes et/ou des secteurs statistiques de moins de 10 logements.

Min. $\mathrm{I}_{\mathrm{j}}=$ valeur minimale.

Tableau 1. Variables constitutives de l'indice socioéconomique

\section{Variables}

Revenu médian (source: INS 2001) (5)

Taux de chômage (source: INS 2001)

Part des noyaux familiaux avec enfant(s) de moins de 18 ans en situation de monoparentalité (source: INS 2001)

Part des ménages isolés au sein des ménages (source: INS 2001)

Part des bénéficiaires d'un revenu d'intégration sociale dans la population en âge d'activité (source: BCSS 2003) (6)

Taux d'emploi (source: INS 2001) 
Détail de l'indice socioéconomique en région urbaine liégeoise (classification en quintiles)

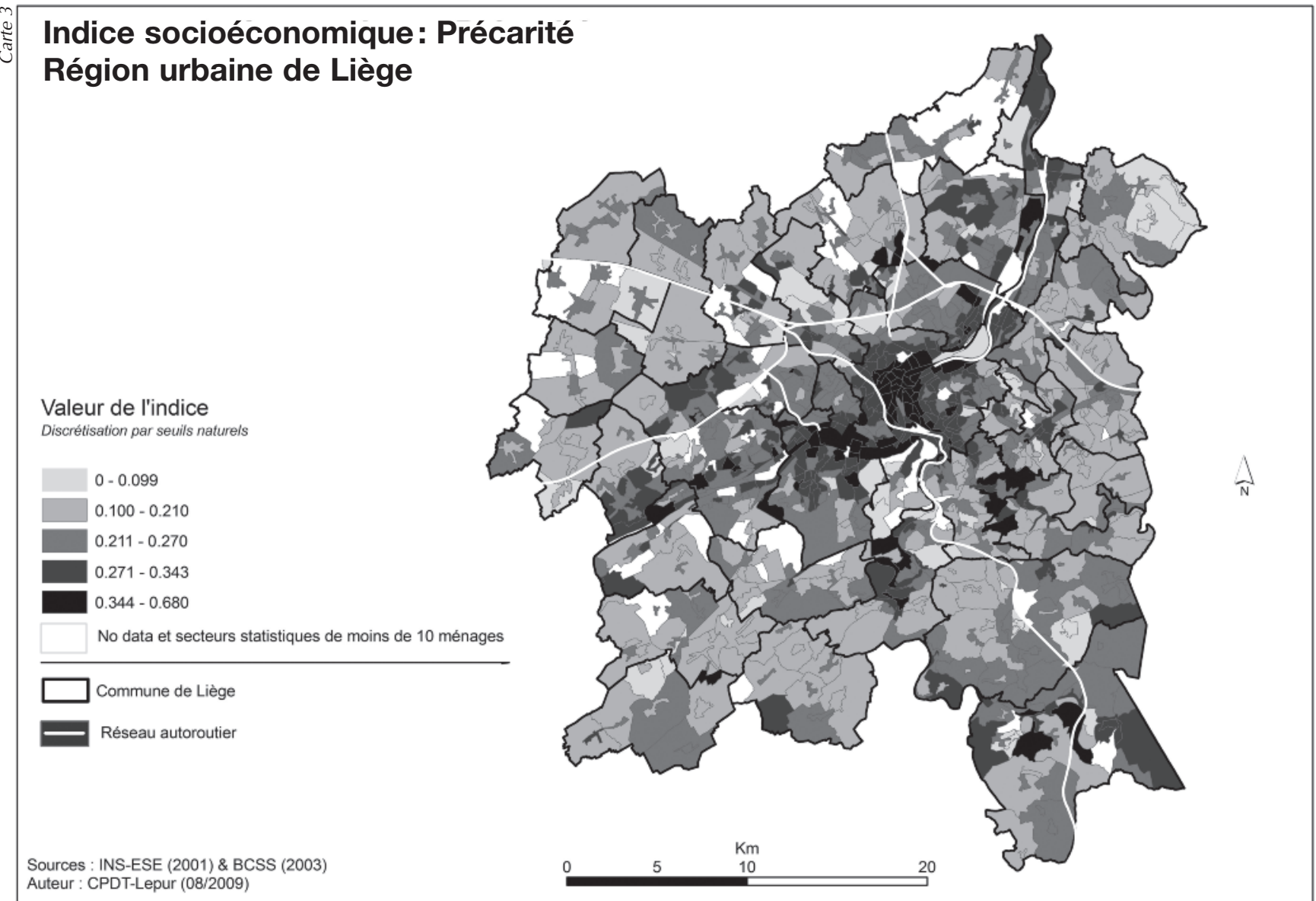

Le secteur statistique (7), qui constitue la zone de référence dans le cadre de l'analyse, correspond plus ou moins à la délimitation physique des quartiers, c'est-à-dire des unités morphologiques constituant la référence de base pour la collecte et la distribution des données statistiques en Belgique. L'objectif consiste ici à identifier les quartiers qui présentent les situations socioéconomiques les plus problématiques (chômage, faibles revenus, etc.).

Deux types de traitements ont été utilisés pour analyser les résultats. Un premier traitement consiste à discrétiser des disparités observées sur la base de seuils naturels. Le second traitement repose sur une classification reposant sur les écarts à la moyenne. Les seuils ont été construits sur la base de la moyenne de l'échantillon (la région urbaine) et de son écart type. Ceci permet de distinguer et de croiser des secteurs statistiques présentant des indices "favorable - moyen - défavorable », selon les quatre dimensions prises en compte: profil socioéconomique, qualité du logement, mobilité et aménités environnementales. Cet artifice sera utilisé dans l'observation d'éventuels renforcements des disparités. Nous n'avons pris en considération que les secteurs statistiques qui présentaient un minimum de dix logements (8), quelle que soit leur superficie. Sont considérés comme favorables les secteurs statistiques dont l'indice est inférieur à la moyenne moins l'écart type et sont considérés comme défavorables les secteurs statistiques dont l'indice est supérieur à la moyenne moins l'écart type.

Les quartiers centraux, localisés le long de l'ancien axe industriel qui s'est développé en fond de vallée, concentrent toujours la plus grande partie des populations précarisées (plus I'indice est élevé, plus la précarité socioéconomique des populations vivant dans le secteur statistique est importante). Cette précarité des populations se mesure ici essentiellement via les 
Synthèse de l'indice socioéconomique en région urbaine liégeoise (classification par écart type)

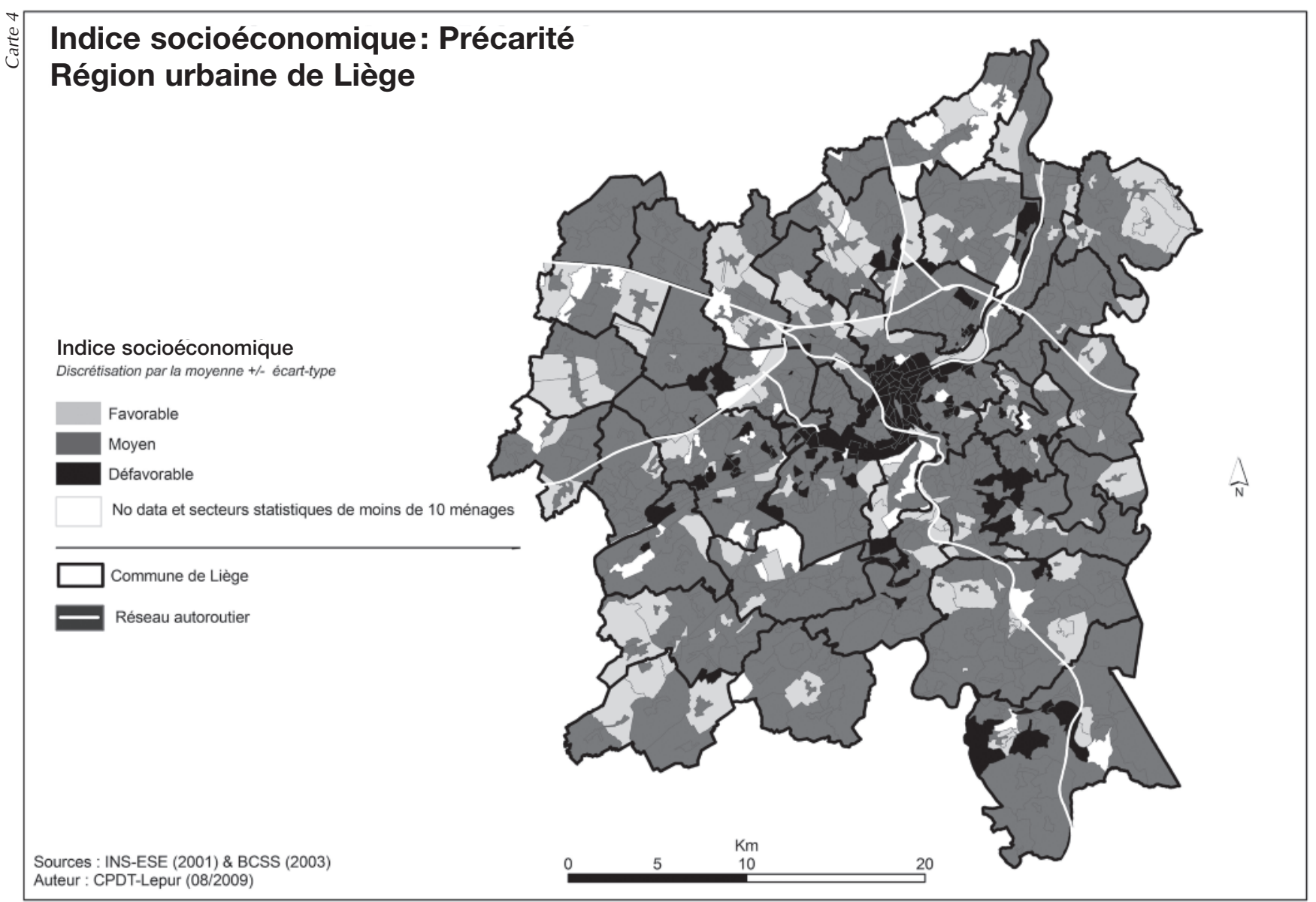

variables envisagées dans l'indice socioéconomique (revenu médian faible, taux de chômage important, part des ménages isolés et monoparentalité, etc.). La plupart des communes plus périphériques concentrent, quant à elles, des populations plus aisées ou issues de la classe moyenne, plus largement propriétaires de leur logement (Vandermotten et al., 2006, p. 43). Cette classification ne doit toutefois pas éluder l'importance des « différentes dimensions sociales, spatiales et institutionnelles de I'organisation urbaine des quartiers dont ne rend pas compte la catégorie de quartier 'pauvre' et quartier 'aisé' » (Bacqué, Fol, 2011 , p. 265), sans oublier les enjeux du vivre ensemble ainsi que du contexte plus général du quartier, dont les réseaux sociaux qui y sont présents.

On constate à la lecture du tableau 2 que ce sont plus de 160000 personnes, soit $25 \%$ de la population de la région urbaine de Liège, qui vivent dans un quartier pour lequel l'indice socioéconomique moyen est considéré comme défavo-

Tableau 2. Répartition de la population selon I'indice socioéconomique

\begin{tabular}{|c|c|c|}
\hline \multicolumn{2}{|c|}{ Tableau 2. Répartition de la population selon I'indice socioéconomique } \\
\hline Indice socioéconomique & Part de la population (en \%) & 33308 \\
\hline Favorable & 5,2 & 440731 \\
\hline Moyen & 69,5 & 160540 \\
\hline Défavorable & 25,3 & 634579 \\
\hline Total & 100 & \\
\hline
\end{tabular}




\begin{tabular}{|c|}
\hline Tableau 3. Variables constitutives de l'indice de qualité du logement \\
\hline Variables \\
\hline Part des logements sans chauffage central (en \%) \\
\hline Part des logements de moins de $35 \mathrm{~m}^{2}(\mathrm{en} \%)$ \\
\hline Logements de plus de 20 ans et logements non transformés entre 91 et $2001(\mathrm{en} \%)$ \\
\hline Part des logements sans double vitrage (en \%) \\
\hline Part des logements sans isolation du toit (en \%) \\
\hline
\end{tabular}

rable. Signalons toutefois que ce type de résultat est susceptible de masquer de fortes disparités internes, des populations aisées pouvant cohabiter avec des populations aux revenus modestes dans un même quartier.

\section{FAIRE LA JONCTION ENTRE LA QUALITÉ DU LOGEMENT ET LES DISPARITÉS SOCIOÉCONOMIQUES}

Nous faisons l'hypothèse que les disparités socioéconomiques sont susceptibles d'être exacerbées ou au contraire atténuées par les qualités intrinsèques du logement. Ces qualités intrinsèques comprennent notamment sa superficie, ses qualités de confort interne et l'accès à un jardin privatif. Cette approche par les qualités intrinsèques du logement a conduit à réaliser un indice de la qualité du logement dans les grandes villes wallonnes sur la base de cinq variables (tableau 3). Cet indice a été réalisé selon la même méthode que celle explicitée précédemment pour l'indice socioéconomique.

À partir de cet indice, on constate que c'est principalement le long de la vallée mosane (voir carte 2) que se concentrent les logements de mauvaise qualité (carte 5). Ce phénomène s'explique notamment par la présence d'un bâti ancien lié au développement lors de l'importante industrialisation (sidérurgie et métallurgie) de la vallée liégeoise aux XIXe et XXe siècles.
L'habitat s'y est développé à proximité directe des unités de production. L'habitat est par contre de meilleure qualité dans les quartiers périphériques de Liège où se concentrent les populations plus aisées et l'urbanisation la plus récente.

L'indice de disparités socioéconomiques a été confronté à celui de la qualité du logement (tableau 4). Les seuils établis pour définir ce dernier ont été calculés en fonction de l'écart type.

On constate ainsi que la qualité du logement joue comme effet « multiplicateur » de la précarisation des ménages dans les quartiers centraux dégradés. Assez logiquement, aucun indice de qualité du logement favorable ne se combine avec un indice socioéconomique défavorable et, à l'inverse, dans $43,8 \%$ des cas, un logement de qualité défavorable se situe dans une zone présentant un indice socioéconomique défavorable. Avec Emelianoff (2010), on peut constater que « la pauvreté est un facteur de vulnérabilité environnementale, déterminant notamment la qualité du logement, qui ne peut plus jouer son rôle de filtre protecteur (isolation thermique, phonique, sismique, confort spatial, qualité des murs, ensoleillement, etc.) et démultiplie au contraire les vulnérabilités» (p. 192). Toutefois, on constate également que plus de $50 \%$ des logements de qualité moyenne sont occupés par des populations classées dans l'in-

\begin{tabular}{|c|c|c|c|c|}
\hline \multirow{2}{*}{ Tableau 4. Répartition des quartiers selon l'indice socioéconomique et l'indice de qualité du logement } \\
\hline \multirow{2}{*}{ Indice socioéconomique } & \multicolumn{4}{|c|}{ Indice de qualité du logement (en \%) } \\
\cline { 2 - 5 } & Favorable & Moyen & Défavorable & Total \\
\hline Favorable & 59,5 & 38,9 & 1,6 & 100 \\
\hline Moyen & 11,1 & 78,6 & 10,3 & 100 \\
\hline Défavorable & 0 & 56,2 & 43,8 & 100 \\
\hline Total & 15,1 & 70,9 & 13,9 & \\
\hline
\end{tabular}


Indice de qualité du logement en région liégeoise

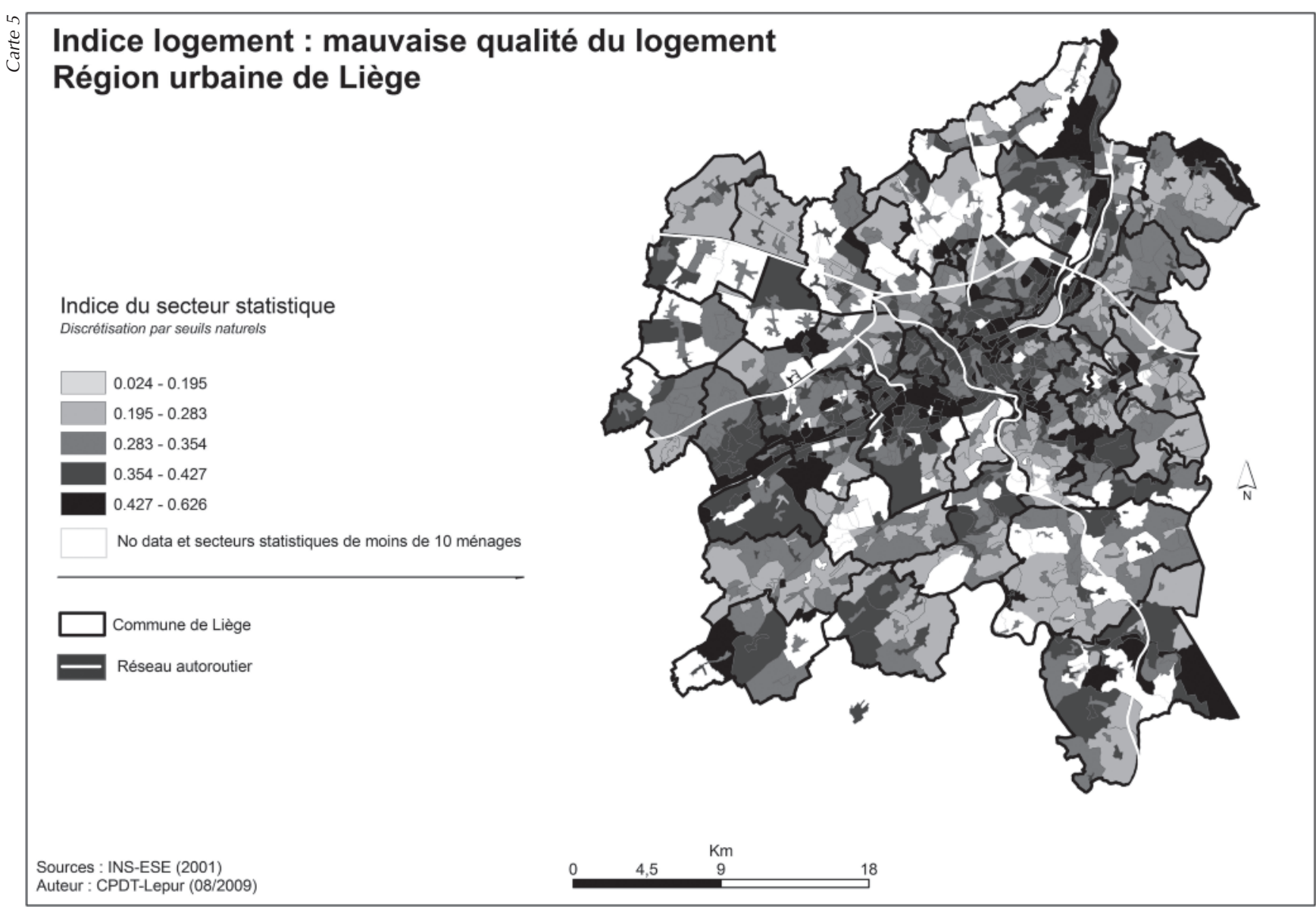

dice socioéconomique défavorable, ce qui permet de nuancer l'idée que les populations les plus précarisées habitent uniquement les logements de mauvaise qualité.

En ce qui concerne la qualité de l'habitat, une enquête menée en 2006-2007 en Wallonie (Carlier et al., 2007) a également permis de déterminer les variations spatiales dans la qualité des logements (9). Il s'agissait d'établir la concordance entre "l'emplacement d'un point de vue urbanistique » du logement, d'une part, et la qualité de celui-ci, d'autre part. Les logements en centres urbains (en bâti continu) ont ainsi été jugés globalement de manière plus négative que dans les autres types de localisation: qualité, salubrité, entretien du quartier, dangers liés à l'équipement électrique ou au gaz, moindre qualité des menuiseries ou encore faible surface habitable. Les services et équipements divers y sont par contre jugés globalement de meilleure qualité.
Afin de préciser ce phénomène de concentration des logements de moins bonne qualité principalement dans les quartiers centraux de la ville (et dès lors le potentiel de renforcement des inégalités socioéconomiques que représente le facteur logement), nous mobilisons trois dimensions explicatives principales: I'histoire économique spécifique de la région liégeoise, les politiques urbaines belge et wallonne et la politique du logement.

\section{L'histoire économique de la région}

Les principaux secteurs d'activité économique ont connu un déclin très important dans la seconde moitié du XXe siècle, contribuant à une paupérisation importante des populations résidant dans le fond de vallée: perte d'emplois, manque de restructuration et d'alternatives économiques. La proximité de ces infrastructures polluantes et génératrices de diverses nui- 
sances contribue à la dégradation du cadre de vie. On peut toutefois s'étonner de la prégnance de cette structure, en particulier lorsqu'on la compare à d'autres régions urbaines, notamment françaises, qui devaient connaître, à partir du début des années 1980, un regain d'intérêt des ménages à revenus moyens et aisés pour les quartiers centraux, bien localisés et au patrimoine urbain attractif (Madore, 1994, p. 22).

\section{Une politique anti-urbaine}

Plusieurs facteurs contribuent à expliquer le constat de la poursuite $d^{\prime}$ 'une politique anti-urbaine en Wallonie depuis I'aprèsguerre. En effet, d'une part, l'évolution des comportements des ménages (décohabitation, augmentation de la part des isolés), les évolutions démographiques (vieillissement, croissance) et les préférences liées au cycle de vie (les jeunes et les personnes peu stables préfèrent la flexibilité du locatif en zone urbaine) ont profondément modifié les modes de vie et d'habitat de la population, d'autre part, en Belgique et en Wallonie, l'idéal de la maison unifamiliale et l'aspiration à la dispersion et à l'isolement urbain, combinés à un manque de capacité planificatrice des autorités ainsi qu'à une très faible régulation des marchés fonciers et à une prégnance du droit de propriété (Halleux, 2005, pp. 247-248) expliquent le phénomène de périurbanisation résidentielle des ménages à revenus moyens ou aisés.

Loopmans et al. (2010) mettent ainsi en exergue dans leur analyse des villes belges que " les politiques belges d'urbanisation ont toujours été caractérisées par un anti-urbanisme fortement enraciné, mettant la priorité sur les développements périphériques, la faible densité en opposition aux quartiers centraux denses. Depuis les années 1960, cette voie a été contestée à de nombreuses reprises. La mixité sociale a dès lors constitué un moyen de pacifier et "coopter" ces défis afin de maintenir l'essence de la politique anti-urbaine. » (p. 182). Ces constats, liés aux priorités en termes de politiques urbaines et de logement, contribuent à expliquer l'ancrage territorial des ménages les plus précarisés au cœur des villes centres, dans un bâti ancien et peu rénové. Ils ne peuvent dès lors que peu influer sur leurs choix résidentiels, en termes de localisation mais également de qualité du logement occupé.

\section{La politique du logement}

En reprenant les formes idéal-typiques de la ségrégation telles qu'elles sont explicitées par Grafmeyer (découlant d'une action organisée et volontaire; indépendante de toute intentionnalité et liée aux inégalités de ressources; " résultat collectif émergeant de la combinaison de comportements individuels discriminatoires »), l'intérêt consiste ici à mettre en exergue « non pas [...] les formes de la ségrégation telles qu'elles peuvent être repérées à travers un jeu de mesures appropriées, mais sur la nature de l'enchaînement causal qui produit ces formes de séparation physique » (Grafmeyer, 1994, p. 105). L'importance d'une analyse en amont des politiques publiques qui sont mises en œuvre et qui contribuent au développement de certaines inégalités sociales et environnementales, notamment dans le domaine du logement, s'avère utile pour expliquer ces phénomènes. Les politiques belges de logement ont réservé des zones considérables à l'habitat au sein des plans d'affectation du sol (équivalents wallons des plans locaux d'urbanisme français) en zones rurales ou périurbaines, afin d'assurer le développement, dans le contexte d'après-guerre, de l'acquisition et/ou construction de maisons unifamiliales, alors que la politique en matière de logement social n'a jamais réellement constitué une alternative ou pu rivaliser avec l'accession à la propriété.

L'accent mis sur l'accession à la propriété privée en Belgique, au moyen de déductions fiscales qui constituent le principal moyen de l'aide au logement, contribue à une relative fixation résidentielle des ménages. Les incitants fiscaux bénéficient en outre surtout aux revenus moyens et élevés (Valenduc, 2008, p. 9), qui ont une capacité d'emprunt suffisante. Ceci explique pour partie l'investissement personnel et financier des populations dans le logement, qui constitue un élément majeur de l'environnement quotidien des ménages. "La satisfaction environnementale serait, avant toute chose, fortement reliée à la dimension affective et identitaire qui s'instaure à l'égard du lieu de vie et en premier lieu à I'habitat » (Faburel, Gueymard, 2009). De plus, ces règles fiscales, combinées à un foncier détenu majoritairement par le secteur privé, contribuent largement à trier les populations en fonction de leur environnement résidentiel.

La concentration des logements locatifs privés dans les centres urbains, plus encore dans les quartiers centraux marqués par un habitat ancien et une forte mixité des fonctions, pose une série d'enjeux en termes de ségrégation sociospatiale. En effet, ce phénomène de concentration des logements locatifs peut renforcer la précarisation des ménages y résidant, conforté par le manque de logements sociaux (qui représentent environ $8 \%$ du parc wallon de logements) - ce qui oblige les demandeurs à se replier sur le logement locatif privé qui 
comble ce manque. La moindre performance énergétique de ce bâti ancien est également problématique, d'autant plus que " les propriétaires [sont] jugés seuls susceptibles de poser des choix pour des équipements liés à la consommation énergétique » (Dozzi et al., 2008). Le rendement énergétique (mise aux normes suite à la directive relative à la performance énergétique des bâtiments) du bâti ancien fait craindre une augmentation des prix de l'immobilier conforme à ces prescriptions et pourrait avoir pour impact une concentration des ménages isolés ou monoparentaux les plus précarisés dans les immeubles les plus anciens et dès lors souvent les moins bien isolés en ville (Vanneste et al., 2007, p. 55), supportant par là même des coûts importants de chauffage. La location de ces logements, temporaire, n'encourage par ailleurs pas leur rénovation et leur embellissement par les populations habitantes. Vandermotten et al. (2006) constatent que "I'insertion des ménages sur le secteur locatif n'en traduit pas moins, en moyenne, un moindre pouvoir d'achat et une moindre capacité d'accumulation patrimoniale, associée non seulement à une qualité de logement souvent inférieure, mais aussi à une plus grande mobilité résidentielle » (p. 12).

En ce qui concerne le statut d'occupation des logements, on constate également que les locataires consacrent une part croissante de leurs revenus à leur loyer et aux charges connexes. En effet, en Wallonie, "la part du logement (loyer, frais de chauffage, d'éclairage, d'eau et les taxes) dans le budget des ménages les plus pauvres est deux fois plus importante que la moyenne wallonne qui atteint déjà un peu plus de $23 \%$ du total du budget des ménages en 2004 » (IWEPS, 2007, p. 130). Les prix des loyers en Wallonie se structurent autour d'une logique centrepériphérie. Les populations habitant les centres de villes, dans un habitat globalement plus ancien qu'en proche périphérie, payent globalement des loyers plus élevés (Vanneste et al., 2007, pp. 144-145).

\section{EN LIEN AVEC LES QUALITÉS EXTRINSÈQUES DU LOGEMENT : LES AMÉNITÉS ENVIRONNEMENTALES}

Suite au constat général fait précédemment, nous allons chercher ici, à travers l'étude de deux aménités urbaines, à mieux comprendre les mécanismes de construction des inégalités environnementales. L'étude du service de l'accessibilité par les alternatives à la voiture (offre en transports en commun), d'une part, et de l'accès aux espaces verts, d'autre part, permettra d'analyser s'ils renforcent les inégalités socioéconomiques pré- cédemment mises en exergue, s'ils contribuent à l'inverse à les compenser ou s'ils sont partiellement déconnectés de celles-ci.

Notre hypothèse consiste en effet à étudier dans quelle mesure les qualités intrinsèques du logement, telles qu'elles ont été explicitées précédemment, constituent le principal vecteur du renforcement des inégalités environnementales dans la région urbaine de Liège ou si d'autres dimensions y contribuent également. Nous avons choisi de nous intéresser à deux types d'aménités urbaines: I'accessibilité par les alternatives à la voiture et l'accès à des espaces verts en milieu urbain. Notre réflexion consiste dès lors à mettre en perspective ces deux types d'aménités urbaines avec la qualité du logement afin d'interroger le postulat de départ consistant à faire du logement, a priori, le principal vecteur du renforcement des inégalités environnementales. Ces deux services nous permettront de montrer la complexité des relations qui se tissent entre ces divers éléments.

\section{L'accessibilité par les alternatives à la voiture}

Dans un premier temps, nous étudions l'offre en transports en commun (et la facilitation de la mobilité douce) qui constitue un enjeu majeur pour les populations les plus précarisées. En effet, le phénomène de captivité potentielle des ménages habitant des zones mal desservies par les alternatives à la voiture pourrait se renforcer au vu de l'évolution du coût des carburants (et du poste qu'il peut représenter pour les ménages contraints $\mathrm{d}^{\prime}$ utiliser régulièrement la voiture individuelle). Ce service urbain, permettant d'assurer une mobilité minimale, a été étudié au moyen d'un indice mesurant l'accessibilité par les alternatives à la voiture (transports en commun et modes lents) (Cornet et al., 2005). L'indice d'accessibilité par les alternatives à la voiture (bus, trains, modes lents) se base sur une modélisation de la distribution spatiale des choix modaux en tout point du territoire wallon (sur la base des données de l'enquête socioéconomique belge de 1991). L'idée qui a présidé à la modélisation est d'étudier le potentiel: le potentiel lié à la densité de population pour les modes lents et le potentiel lié à l'offre pour les transports en commun (fréquence de la desserte et distance à un arrêt ou à une gare). Sur la base de ce modèle, chaque secteur statistique s'est vu attribuer une valeur unique par généralisation/agrégation.

Ce service est central car il permet aux populations (y compris les plus défavorisées mais également jeunes) de se déplacer, notamment pour le motif emploi ou scolaire, et offre une 
Tableau 5. Répartition des quartiers selon l'indice socioéconomique et l'indice de mobilité

\begin{tabular}{|c|c|c|c|c|}
\hline \multirow{2}{*}{ Indice socioéconomique } & \multicolumn{4}{|c|}{ Indice de mobilité (en \%) } \\
\hline & Favorable & Moyen & Défavorable & Total \\
\hline Favorable & 0 & 74,6 & 25,4 & 100 \\
\hline Moyen & 9,2 & 79,9 & 10,8 & 100 \\
\hline Défavorable & 54,9 & 42,5 & 2,6 & 100 \\
\hline Total & 14,5 & 74,1 & 11,3 & 100 \\
\hline
\end{tabular}

alternative dans des villes congestionnées par l'automobilité. «La mobilité - largement considérée aujourd'hui comme une valeur positive et comme une ressource sociale importante pose problème (en termes de coût ou de difficulté) » (Coutard et al., 2002, p. 3). Notre étude a fait le choix d'analyser la mobilité via le service transports en commun, faisant abstraction, d'une part, des motifs de déplacements et, d'autre part, de l'utilisation réelle qui en est faite par les populations pour mettre en exergue la mobilité potentielle - (in)égalités des chances - (et non effective) dont elles disposent. Une analyse des comportements de mobilité, notamment via une approche qualitative, permettrait de compléter cette étude. En effet, l'éventail des " possibles » dépend de toute une série de considérations dont la mobilité mais aussi de considérations économiques, familiales, etc. (Wenglenski, 2010, p. 125).

L'accessibilité par les alternatives à la voiture est la plus importante non seulement dans les quartiers centraux de la ville (réseau très dense) mais également en proche périphérie. À l'inverse, la plupart des communes périurbaines voire situées plus loin du centre, hormis les communes situées en fond de vallée, sont extrêmement dépendantes de la voiture individuelle.

On constate ainsi que la mobilité joue, à Liège, plutôt comme une forme de compensation des inégalités sociales que comme renforcement de celles-ci (tableau 5). Remarquons cependant qu'un certain nombre d'activités économiques et commerciales et, dès lors, de lieux d'emploi se sont déplacés vers les périphéries de Liège, ce qui les rend relativement moins accessibles par les alternatives à la voiture. Le phénomène de centralité, caractérisé par la concentration des populations défavorisées dans des zones urbaines denses bien connectées aux réseaux de transports en commun, constitue un phénomène spécifique à la Wallonie. En France, un modèle plutôt inver- se domine, même si « en termes de mobilité et de dépendance à l'automobile, les contextes territoriaux en grande couronne sont fortement contrastés» (Motte-Baumvol, 2009, p. 55). Plusieurs nuances peuvent être apportées afin de mieux appréhender les phénomènes de la mobilité quotidienne des ménages: l'absence d'automobile, l'importance des réseaux sociaux et familiaux de proximité, la densité de services et de commerces, etc. En effet, « l'exclusion d'un nombre plus important de ménages par de fortes disparités de mobilité est plus certainement à aller chercher du côté des ménages motorisés » (ibid. p. 65). Une étude complémentaire sur la région liégeoise pourrait permettre, au-delà d'une analyse basée sur l'offre potentielle en alternatives à la voiture, de distinguer ces situations entre ménages motorisés et non-motorisés.

Faburel et Geymard (2008) constataient ainsi qu'on ne peut pas toujours conclure à une «suprématie environnementale du groupe sociourbain aisé » (p. 170). Ce constat peut aussi être fait dans le cas liégeois où le cumul des inégalités sociales et environnementales n'est pas systématique dans l'accès à certains services urbains, comme l'offre en transport alternatif à la voiture.

\section{L'accès aux espaces verts}

L'accessibilité aux espaces verts a, dans un second temps, été étudiée au moyen d'un indice spécifique. Nous retrouvons ici une conception plus « traditionnelle » de l'inégalité environnementale comme possibilité d'accès à la nature - espaces publics, jardins, forêts, etc. - partant de I'hypothèse que les populations défavorisées seraient également plus lésées en ce qui concerne cette accessibilité. La qualité « environnementale » a ainsi un impact sur les paysages, I'attractivité, les prix immobiliers ainsi que le cadre de vie général d'un quartier. 


\section{Accessibilité aux espaces verts (12)}

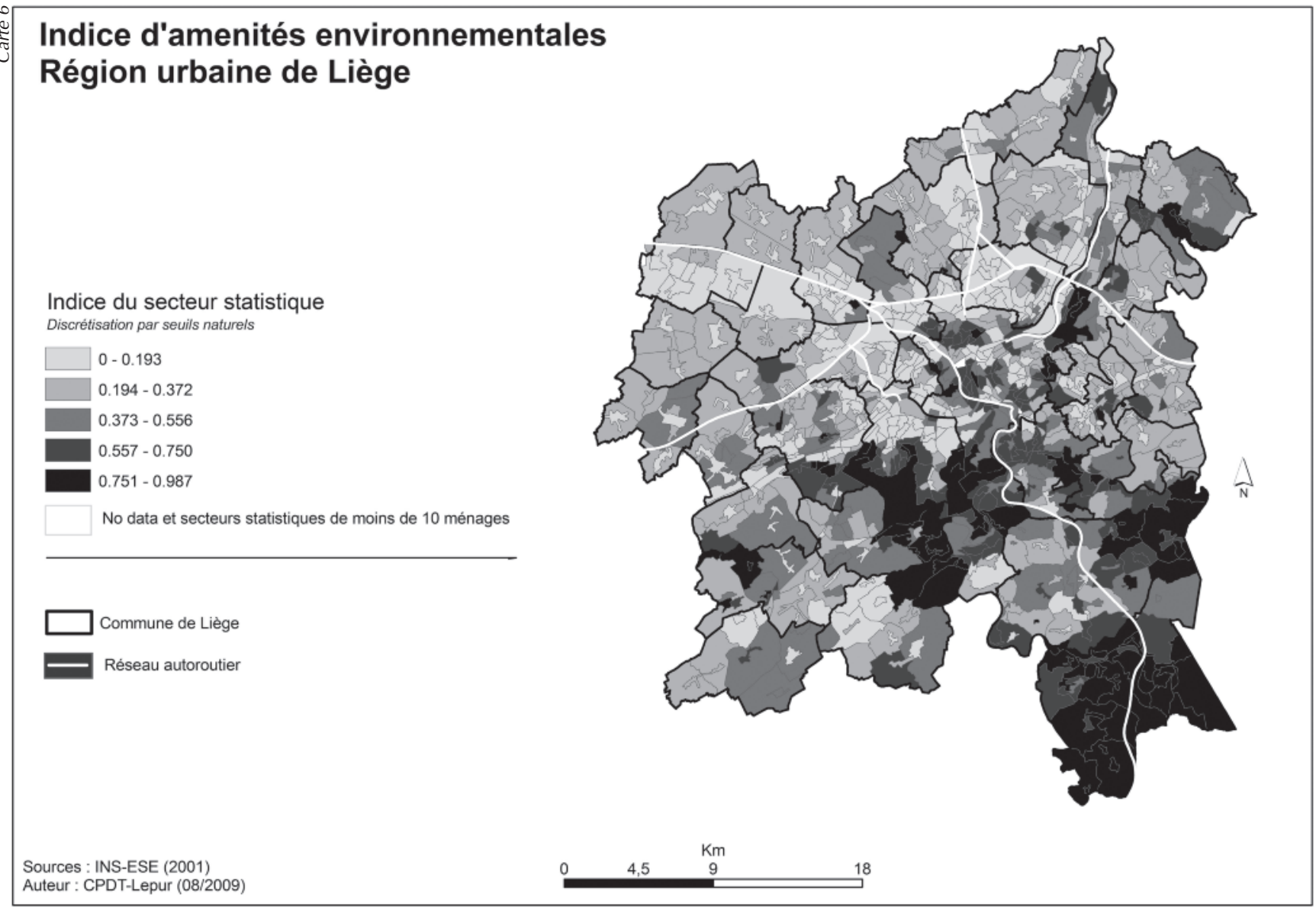

Deux sous-indices ont été développés: la composante verte paysagère totale et l'accessibilité aux espaces verts publics. Cet indice d'accès aux espaces verts indique aussi bien des parcs que des espaces agricoles ou des massifs forestiers. Il peut de ce fait s'agir d'espaces de très grande taille. Leur accessibilité physique n'est donc pas garantie, même si leur proximité peut constituer une forme d'aménité, ne fût-ce que sur le plan visuel. Ce choix de travailler sur la base de deux sous-indices nous a permis de prendre en considération deux manières de concevoir les espaces verts: d'une part, une approche brute (10) qui se base sur l'occupation du sol, intégrant toutes les surfaces qui ne sont pas urbanisées au sens large et, d'autre part, une approche stricte des espaces verts considérés comme des espaces aménagés (11). Une plus grande importance a toutefois été donnée à l'approche stricte à laquelle on a octroyé un poids double.
La première composante constitue donc une estimation brute de l'aménité des quartiers, c'est-à-dire des espaces verdurisés de tout type sur la base de la carte d'occupation des sols de Wallonie. La seconde, l'accessibilité aux espaces verts, a été étudiée sur la base du classement des espaces verts en fonction de leur rayonnement: un rayon de $150 \mathrm{~m}$ pour les petits espaces et un rayon de $400 \mathrm{~m}$ pour les espaces dont les dimensions sont supérieures à un hectare, comme cela a été développé dans le cadre du plan flamand AMINAL (1993). À la différence du précédent, les espaces verts considérés ici sont accessibles et aménagés. Un indice d'aménités environnementales regroupant ces deux composantes a dès lors été produit qui calcule la moyenne des parts de couverture d'accessibilité aux espaces verts publics et de la composante verte paysagère totale (avec un poids double donné aux valeurs d'accessibilité). Ces indices n'intègrent cependant pas la qualité des espaces ni leur acces- 
Tableau 6. Répartition des quartiers selon l'indice socioéconomique et l'indice d'aménités environnementales

\begin{tabular}{|c|c|c|c|c|}
\hline \multirow{2}{*}{ Indice socioéconomique } & \multicolumn{4}{|c|}{ Indice d'aménités environnementales (en \%) } \\
\hline & Favorable & Moyen & Défavorable & Total \\
\hline Favorable & 23,8 & 64,3 & 11,9 & 100 \\
\hline Moyen & 19,7 & 64,6 & 15,7 & 100 \\
\hline Défavorable & 28,1 & 54,9 & 16,9 & 100 \\
\hline Total & 21,3 & 63,2 & 15,4 & 100 \\
\hline
\end{tabular}

sibilité réelle. En effet, la présence de barrières physiques empêchant un accès effectif à ces espaces n'a pas pu être intégrée à I'indice.

L'indice d'aménités environnementales ne reproduit pas un schéma centre-périphérie, comme on pourrait le supposer. En effet, certains parcs urbains permettent d'offrir une réelle accessibilité aux espaces verts en ville tandis qu'en milieu rural ou périurbain, certaines zones ne sont pas accessibles au public (zones agricoles, par exemple). Malgré l'accès moins aisé des populations présentant un indice socioéconomique plus faible, nous ne pouvons conclure à une dichotomie entre population aisée vivant dans un environnement de haute qualité et populations défavorisées hors de portée des espaces verts et de récréation. Ce deuxième service urbain ne permet dès lors pas de conclure à un renforcement systématique des inégalités socioéconomiques (mises en exergue par l'indice socioéconomique) par l'accès à la nature. II démontre de multiples réalités et une relative déconnexion entre ces deux aspects, comme l'illustre la carte 6, en particulier dans le centre-ville et la zone nord de la ville.

Au-delà d'une lecture " physicaliste » et technique de l'environnement, pour reprendre les termes de Faburel (2008, p. 120), pourrait également s'ouvrir une réflexion relative à une approche plus subjective de la qualité d'environnement et du cadre de vie, centrée sur les idées et les perceptions des populations en place. La capacité de mobilisation des populations locales face aux enjeux environnementaux est également appréhendée aujourd'hui comme une des facettes importantes des inégalités environnementales ainsi que du mouvement de la justice environnementale. Ces aspects sont cependant encore très peu étudiés en Wallonie et mériteraient des analyses complémentaires.

\section{CONCLUSION}

L'objet de cette étude était d'identifier le principal vecteur du renforcement des inégalités socioéconomiques et de la précarité des ménages dans la région urbaine de Liège en proposant un croisement entre un indice de la qualité du logement, d'une part, et des indices d'accès aux aménités environnementales résidentielles (mobilité et espaces verts), d'autre part. Notre hypothèse de base consistait dès lors à postuler que les qualités intrinsèques du logement, envisagées comme variable environnementale principale, constituent le facteur majeur d'accentuation de la ségrégation socioéconomique dans les villes en Wallonie.

De manière générale, il ressort de la comparaison des volumes de population exposée à des mécanismes de double précarité que le logement reste, en Wallonie, en comparaison des deux autres aménités étudiées, un instrument important du renforcement des disparités socioéconomiques, bien avant I'accessibilité aux espaces verts ou l'accessibilité aux transports en commun. Ceci vient appuyer notre choix méthodologique consistant à considérer la qualité environnementale sur la base d'une analyse du continuum allant de l'échelle du logement à celle de la région urbaine. Le postulat de départ nous ayant conduits à interroger l'impact des qualités intrinsèques du logement sur le renforcement des inégalités s'avère dès lors déterminant pour comprendre un des mécanismes majeurs de la ségrégation sociospatiale vis-à-vis des populations les plus défavorisées dans la zone urbaine de Liège.

La mauvaise qualité intérieure du logement n'explique bien évidemment pas de manière causale et unilatérale la paupérisation des populations qui, à son tour, a également un impact sur la qualité du logement et ses attributs. Le logement peut éga- 
lement être envisagé comme un lieu d'identité et de refuge pour les ménages.

Par contre, la desserte en transports en commun traduit, pour sa part, une forme de compensation territoriale - l'offre étant concentrée essentiellement dans les quartiers affichant les indices socioéconomiques les plus défavorables. En ce qui concerne les espaces verts accessibles au public, on constate que cette dimension n'est plus structurée sur un rapport centrepériphérie mais doit se lire à l'échelle plus large de la région urbaine liégeoise.

S'éloigner d'une approche plus traditionnelle liée à l'étude des pollutions, de la proximité aux installations polluantes ou à l'accès à des aménités (même si notre étude est complétée par une analyse relative à certaines de ces aménités) nous a permis de mettre en avant un facteur parfois largement sous-estimé dans les études relatives aux inégalités environnementales: la qualité du logement comme élément central du cadre de vie des populations.

La prise en considération de ces inégalités, aujourd'hui absente des politiques publiques destinées aux territoires urbains en Wallonie, pourrait à l'avenir contribuer à la définition de priorités pour ces territoires largement délaissés par les populations aisées ainsi que par les secteurs d'activités économique et commerciale. Le concept d'inégalités environnemen- tales renforce le besoin d'une action coordonnée à une échelle territoriale plus large que la commune. Il invite également à une réflexion sur les mécanismes de la ségrégation sociospatiale, sur la justice territoriale et met en lumière l'ambivalence de la question vis-à-vis d'autres services urbains majeurs que sont I'accessibilité par les alternatives à la voiture et l'accès à la nature.

Zoé Lejeune est doctorante IPRA (IWEPS PhD Research Activity) au Département de Science Politique (European Studies Unit) et au Lepur (Centre de Recherche en Science de la Ville, du Territoire et du Milieu Rural) à l'Université de Liège. Politologue de formation, elle travaille depuis plusieurs années sur les thématiques de l'aménagement du territoire, de l'urbanisme et de l'environnement zoe.lejeune@ulg.ac.be

Géographe de formation, Thomas Chevau a travaillé comme attaché de recherche au Lepur pendant deux années et a ensuite été conseiller au Cabinet du Ministre wallon de l'Environnement, de I'Aménagement du territoire et de la Mobilité. II est aujourd'hui coordinateur de projets à la SPI (Agence de Développement économique pour la province de Liège).

Jacques Teller est chargé de cours à I'Université de Liège, directeur scientifique du LEMA (Local Environment Management \& Analysis) et président du Lepur (Centre de recherche en Sciences de la Ville, du Territoire et du Milieu rural). Il travaille sur les questions de gouvernance et de prise de décision en matière d'aménagement du territoire ainsi que sur les modèles d'analyse de la forme urbaine. II a coordonné les recherches européennes SUIT, APPEAR et PICTURE et a été le président de l'Action COST C21, dédiée aux ontologies urbaines. jacques.teller@ulg.ac.be

RemerCiements : Cette étude a été financée par la Conférence Permanente du Développement Territorial (CPDT) dans le cadre des recherches relatives à la Politique de la ville et à l'actualisation du SDER.

\section{NOTES}

(1) Le ménage en état de précarité dispose globalement de moins de $10000 €$ de revenus annuels imposables.

(2) Voir notamment à ce sujet: De Spiegellaere, 2007

(3) En Grande Bretagne: voir Lucas et al., 2004; Brainard et al., 2002. En France: voir les travaux de Faburel, Emelianoff, Theys, Laigle et Oehler ainsi que les publications du MEDD.

(4) II ne s'agit pas d'une limite administrative.

(5) L'INS (Institut national de la statistique) s'appelle aujourd'hui DGSIE: Direction Générale Statistique et Information Économique.

(6) La BCSS est la Banque centrale de la sécurité sociale belge.

(7) Il est officiellement défini par la DGSIE (Direction générale Statistique et Information économique) comme «l'unité territoriale de base qui résulte de la subdivision du territoire des communes et anciennes communes par l'Institut national de
Statistique pour la diffusion de ses statistiques à un niveau plus fin que le niveau communal » (Jamagne, 2012).

(8) La variabilité de l'échantillon, mesurée par l'écart type, a conduit à opter pour un seuil de dix logements, représentatif.

(9) Cette enquête sur la qualité de l'habitat n'a toutefois pas pu être utilisée dans le cadre de notre indice relatif à la qualité du logement car elle était basée sur des entretiens menés dans 6000 logements, ce qui ne permettait pas de spatialiser les données à l'échelle du secteur statistique.

(10) Voir à ce sujet: Fouchier, 1998.

(11) Pour une définition de l'espace vert " aménagé », se référer à la notion telle qu'elle est explicitée dans Merlin et Choay, 1996.

(12) Cet indice a été calculé de la même manière que les autres indices présentés précédemment. 


\section{BibliograPHIE}

AMINAL, 1993, Lange Termijnplanning Groenvoorziening, Boekdeel 1. Onderzoek uitgevoerd door Mens en Ruimte vzw en VVOG vzw. Ministerie van de Vlaamse Gemeenschap, Brussel

BACQuÉ M.-H., Fol S., 2011, "Politiques de mobilité résidentielle et de déségrégation: une analyse critique », Regards croisés sur l'économie, vol.1, n9, pp. 261-271.

Blanchon D., Moreau S. et Veyret Y., 2009, "Comprendre et construire la justice environnementale », Annales de la Géographie, n665-666, pp. 35-60.

BOUlder Wenz P.S., 1988, Environmental Justice, New York, State University of New York Press

Brainard J. S., Jones A. P., Bateman I. J., Lovett A. A., Fallon P. J., 2002, "Modelling environmental equity: access to air quality in Birmingham UK", Environmental and Planning A, 4, pp. 695-716.

Bullard R., 1990, Dumping in Dixie: Race, Class, and Environmental Quality, Boulder, Co, Westview Press

Carlier P., Fontaine S., Monnier B., 2007, « La qualité de I'habitat wallon: synthèse des résultats de l'enquête », Les Échos du Logement, Région wallonne, n4, pp. 1-15.

Cornet Y., Daxhelet D., Halleux J.-M., KlinkenberG A.-C., LAmBotte J.-M., 2005, "Cartographie de l'accessibilité par les alternatives à la voiture ». In: Witlox F., Van Acker V. (dir.), Les journées cartographiques belges. Mobilité, Société et Environnement en Cartes, Zelzate, Belgique, DCL Print \& Sign, pp. 355-364.

Coutard O., Dupuy G., Fol S., 2002, « La pauvreté périurbaine: dépendance locale ou dépendance automobile? ", Espaces et Sociétés, n¹08-109, pp. 155-176.

De Spiegelaere M., 2007, "Inégalités sociales, logement et santé ». In: Cornut P., Bauler T., Zaccaï E., Environnement et inégalités sociales, Bruxelles, Éditions de I'Université de Bruxelles, Aménagement du territoire et Environnement, pp. 117-125.

De Decker P., Laureys J., 2007, "Le marché du logement se polarise-t-il à Bruxelles et en Wallonie? ", Les Échos du Logement, Région wallonne, $\mathrm{n}^{\circ} 1, \mathrm{pp} .1-16$.

DOzZI J., LeNNERT M., WALLENBORN G., 2008, «Inégalités écologiques: analyse spatiale des impacts générés et subis par les ménages belges », Espaces, populations, sociétés, $\mathrm{n}^{\circ} 1$, pp. 127-143.

Dujardin S., Labeeuw F.-L., Melin E., Pirart F., Teller J., 2010, Thème 2B. Structuration du territoire pour répondre aux objectifs de réduction des émissions de gaz à effet de serre, Rapport final de la subvention CPDT 2009-2010, octobre

EMELIANOFF C., 2010, " Les inégalités écologiques et environnementales, au point de rupture d'un modèle de développe- ment?", Urbia - Les cahiers du développement urbain durable, n¹1, décembre, pp. 181-202.

EPA (Environmental Protection Agency), 2004, Toolkit for Assessing Potential Allegations of Environmental Injustice, Report, EPA, Washington.

FABurel G., 2008, « Les inégalités environnementales comme inégalités de moyens des habitants et des acteurs territoriaux: pour que l'environnement soit un facteur réel de cohésion urbaine », Espaces, populations, sociétés, $\mathrm{n}^{\circ} 1$, pp. 111-126.

Faburel G., Gueymard S., 2009, "Inégalités environnementales, inégalités sociales ", Les Cahiers de I'IAU-Île-deFrance, $\mathrm{n}^{\circ} 152$, novembre, Composer avec l'environnement, pp. 22-24.

FABurel G., GueYMARD S., 2008, «Inégalités environnementales en région île-de-France: le rôle structurant des facteurs négatifs de l'environnement et des choix politiques afférents », Espaces, population, sociétés, n¹, pp. 159-172.

FOUCHIER V., 1998, Les densités urbaines et le développement durable: le cas de l'île-de-France et des villes nouvelles, Paris, Édition du SGVN

GRAFMEYER Y., 1994, "Regards sociologiques sur la ségrégation ». In: Brun J., Rhein C. (dir.), La ségrégation dans la ville, Paris, L'Harmattan, Habitat et Société, pp. 85-118.

GueYmard S., 2004, "Le rôle des espaces verts dans les arbitrages résidentiels des ménages: une étude exploratoire dans trois communes du Val-de-Marne », mémoire de DEA, Institut $\mathrm{d}^{\prime}$ Urbanisme de Paris

Halleux J.-M., 2005, Structuration spatiale des marchés fonciers et production de l'urbanisation morphologique: application à la Belgique et à ses nouveaux espaces résidentiels, Thèse de doctorat, 24 juin 2005, Université de Liège, Département de Géographie

IWEPS, 2007, Rapport sur la cohésion sociale en Région wallonne: Volet Statistique, Une Collection de la DIIS Direction interdépartementale de l'Intégration sociale du Ministère de la Région wallonne

Jamagne P., 2012, Secteurs statistiques: Vade-mecum, DGSIE (Direction générale Statistique et Information Économique), En ligne: http://statbel.fgov.be/fr/binaries/Secteur\%20statFR_tcm326-174181.pdf, consulté le 26 septembre 2012

JONCKHEER P., 2007, "Indoor pollution et inégalités sociales: analyse de la littérature scientifique ». In: Cornut P., Bauler T., Zaccaï E., Environnement et inégalités sociales, Éd. de I'Université de Bruxelles, Aménagement du territoire et Environnement, Bruxelles, pp. 127-133.

Laigle L., Oehler V., 2004, Les enjeux sociaux et environnementaux du développement urbain, la question des inégali- 
tés écologiques, Rapport final, Paris, CSTB

Loopmans M., De Decker P., Kesteloot C., 2010, "Social Mix and Passive Revolution. A Neo-Gramscian Analysis of the Social Mix Rhetoric in Flanders, Belgium", Housing Studies, vol. 25, n², mars, pp. 181-200.

lucas K., Walker G., Eames M., fay H., Poustie M., 2004, Environment and Social justice: rapid research and evidence review, Final report, SDRN, DEFRA

MAdORE F., 1994, "Marchés immobiliers des espaces centraux et dynamiques sociales de moyennes villes françaises ». In: Chevalier J., Peyon J.-P. (dir.), Au centre des villes: dynamiques et recompositions, Paris, L'Harmattan, Géographie sociale, pp. 15-44.

MATSUOKA M., 2001, "The emergence of the environmental justice movement and its challenges to planning", Critical Planning Journal, n8, pp. 4-14.

MerLin P., ChOAY F. (dir.), 1996, Dictionnaire de I'urbanisme et de l'aménagement, Paris PUF, 2e éd.

Motte-BAumvol B., 2009, "Les populations périurbaines face à I'automobile en grande couronne francilienne ", Norois, vol. 4, n²05, pp. 53-66. En ligne:

http://norois.revues.org/1251, consulté le 25 septembre 2012

TelLer J. (dir.), 2009, Expertise Politique de la ville. Annexes au rapport final de la subvention 2008-2009, Conférence Permanente du Développement Territorial, Septembre, Wallonie.

THEYS J., 2002, « L'approche territoriale du 'développement durable', condition d'une prise en compte de sa dimension sociale », Développement durable et territoires, Dossier 1 : Approches territoriales du Développement Durable. Mis en ligne le 23 septembre 2012 :

http://developpementdurable.revues.org/1475
THEYs J., 2007, « Pourquoi les préoccupations sociales et environnementales s'ignorent-elles mutuellement? Un essai $\mathrm{d}^{\prime}$ interprétation à partir du thème des inégalités écologiques ». In: Cornut P., Bauler T., Zaccaï E. (dir.), Environnement et inégalités sociales, Éditions de I'Université de Bruxelles, Aménagement du territoire et environnement, pp. 23-35.

VAlENDUC C., 2008, " Les incitations fiscales en faveur du logement », Les Échos du Logement, n², pp. 1-16.

Van der Haegen H., Van Hecke E., Juchtmans G., 1996, "Les régions urbaines belges en 1991 », Études statistiques, $\mathrm{n}^{\circ} 104$, pp. 3-42.

Vandermotten C., Marissal P., Van Hamme G., Kesteloot C., Slegers K, Vanden Broucke L., Ippersiel B., De Bethune S., NAIKEN R., 2006, Analyse dynamique des quartiers en difficulté dans les régions urbaines belges, SPP Intégration Sociale - Politique des Grandes Villes

Vanneste D., Thomas I., GoOssens L., 2007, Le logement en Belgique, enquête socioéconomique 2001, monographies, SPF Économie, P.M.E., Classes moyennes et Énergie, Bruxelles, Direction générale Statistique et Information économique

Wenglenski S., 2010, "Accès à l'emploi: champ des possibles contre champ des pratiques ». In: Massot M.-H. (dir.), Mobilités et modes de vie métropolitains: les intelligences du quotidien, Paris, Éditions l'œil d'Or, critiques \& cités, pp. 121-137.

ZACCAï E., 2007, « Les inégalités écologiques, facteurs ou non d'aggravation des disparités sociales », Séminaire d'animation des recherches du programme, Programme de recherche Politiques territoriales et développement durable, Synthèse de la séance thématique 8, 16 janvier, pp. 3-4. 